\title{
El rol del estudiante en los ambientes educativos mediados por las TIC*
}

\author{
Paul Andrés Rugeles Contreras ${ }^{\star *}$, Beatriz Mora González ${ }^{\star * *}$, Piedad María Metaute Paniagua***
}

\begin{abstract}
"Las TIC son una nueva cultura, son un nuevo lenguaje (Logan), son un nuevo ambiente (McLuhan), una nueva piel de la cultura (de Kerckhove), que cambian todas las reglas y que reinventan de cabo

a rabo (Weinberger), no solo qué es aprender, sino qué es vivir en un entorno en donde ya no hay escasez sino sobreabundancia de información, en donde el aprendizaje es cada vez mas heterónomo, en donde el mundo escolar pierde el monopolio cognitivo, en donde los profesionales de la transmisión seriada ven de pronto desvalorizadas sus competencias y habilidades".
\end{abstract}

Alejandro Piscitelli (2012)

\section{Resumen}

En el presente artículo se hace referencia a los resultados del trabajo investigativo "Caracterización de experiencias significativas mediadas por las $\mathrm{TIC}$, en Educación Superior virtual", en el que se identificaron factores potenciadores para la configuración de este tipo de experiencias, como la autodisciplina, el autoaprendizaje, el análisis crítico y reflexivo, y el trabajo colaborativo, Rugeles, Mora, Metaute, (2013). Estos factores son abordados como conceptos clave en el rol del estudiante en los ambientes educativos mediados por las TIC, donde especialmente, se esboza un panorama en el que los estudiantes asumen roles que los comprometen, aún más, con su propio proceso de aprendizaje. Dichos roles, en conjunto, pretenden fortalecer las capacidades subjetivas e intersubjetivas de los estudiantes, como seres sociales comprometidos que pueden contribuir con el desarrollo social de las comunidades en las que se encuentran inmersos.

Palabras clave: rol del estudiante virtual, auto disciplina, auto aprendizaje, trabajo colaborativo, análisis crítico y auto reflexivo, herramientas TIC.

\section{The student's role in the educative environments mediated by IT}

\begin{abstract}
This article references the results obtained in the research work "Caracterización de experiencias significativas mediadas por las TIC, en Educación Superior virtual" (Characterization of significant experiences mediated by IT in virtual higher education), in which factors that potentiate the configuration of such experiences, such as selfdiscipline, self-learning, critical and reflective analysis and collaborative work were identified (Rugeles, Mora, Metaute, 2013). These factors are approached as key concepts in the role of students in educative environments mediated by IT, where, in a especial way, appears a scope in which students assume roles that make them commit even more with their learning process. Such roles, together, aim to strengthen the subjective and inter subjective capacities of students as committed social beings who can contribute to the social development of their communities.
\end{abstract}

\footnotetext{
* $\quad$ Artículo de reflexión derivado del trabajo de investigación titulado "Caracterización de experiencias significativas mediadas por las TIC en educación superior virtual”, realizado para optar el título de magíster en Educación y Desarrollo Humano, del convenio Universidad de Manizales-CINDE, Autores: Paul Andrés Rugeles Contreras, Beatriz Mora González y Piedad María Metaute Paniagua.

** Licenciado en Matemáticas y Física, ingeniero industrial, especialista en Gerencia Financiera, magíster en Educación y Desarrollo Humano. Email: paul.rugeles@ceipa.edu.co, Docente de la Fundación Universitaria CEIPA (Sabaneta-Colombia).

*** Ingeniera industrial, especialista en Métodos Administrativos y de Producción, magíster en Educación y Desarrollo Humano, E-mail: bmoragonzalez@gmail.com, Docente de la Universidad de San Buenaventura (Medellín-Colombia).

**** Ingeniera de Sistemas. Especialista en Finanzas. Magíster en Educación y Desarrollo Humano. E-mail: piedad.metaute@remington. edu.co-pmetaute@gmail.com Docente de la Corporación Universitaria Remington-Uniremington (Medellín-Colombia).
}

Autor para correspondencia: Paul Andrés Rugeles Contreras, e mail: paul.rugeles@ceipa.edu.co.

Artículo recibido: 18/06/2015; artículo aprobado: 18/08/2015. 
Key words: role of virtual students, self-discipline, self-learning, collaborative work, critical and selfreflective analysis, IT tools.

\section{O papel do estudante nos ambientes educativos mediados pelas TICs}

\section{Resumo}

No presente artigo se faz referência aos resultados do trabalho investigativo "Caracterização de experiências significativas mediadas pelas TICs, em Educação Superior virtual", no que se identificaram fatores potenciadores para a configuração deste tipo de experiências, como a autodisciplina, o autoaprendizagem, a análise crítica e reflexiva, e o trabalho colaborativo, Rugeles, Mora, Metaute, (2013). Estes fatores são abordados como conceitos chaves no papel do estudante nos ambientes educativos mediados pelas TICs, onde especialmente, esboça-se um panorama no que os estudantes assumem papéis que os comprometem, ainda mais, com seu próprio processo de aprendizagem. Ditos papéis, em conjunto, pretendem fortalecer as capacidades subjetivas e intersubjetivas dos estudantes, como seres sociais comprometidos que podem contribuir com o desenvolvimento social das comunidades nas que se encontram imersos.

Palavras chave: papel do estudante virtual, autodisciplina, autoaprendizagem, trabalho colaborativo, análise crítica e auto reflexivo, ferramentas TICs.

\section{Introducción}

Las tecnologías de información y comunicación (TIC de aquí en adelante) han hecho su incursión en todos los campos de acción del ser humano. Su principal propulsor ha sido Internet, al que Piscitelli $(2002,207)$ define como "el primer medio masivo de la historia que permitió una horizontalización de las comunicaciones, una simetría casi perfecta entre producción y recepción, alterando en forma indeleble la ecología de los medios".

Si bien los inicios de Internet estuvieron asociados a la creación de una red llamada Arpanet, de uso exclusivo militar en los años sesenta con el objetivo de tener información desde cualquier punto del país (Estados Unidos) en caso de ataques rusos, dicho concepto cambia en los años 90 debido a su expansión en todo el mundo a través de miles de redes interconectadas que conformaron una gran red de redes. Esta red favoreció el intercambio, a gran escala, de datos e información. Lamarca (2011) expone que dicha versatilidad es atribuida a Tim Gerners-Lee quien combinó dos tecnologías existentes: el hipertexto y el protocolo de comunicaciones de Internet -IP, creando así un nuevo modelo de acceso (World Wide Web) que hace posible la navegación de cualquier usuario a través de la red sin ninguna dificultad.
La evolución de la World Wide Web ha permitido transformar y dinamizar los sitios y páginas web, potenciar los navegadores, mejorar herramientas multimediales (vídeo, imagen, texto, sonido), ofrecer servicios transaccionales, dinamizar las bodegas de datos, servicios on line, entre otros. Por esta razón, sehaconvertidoenelmedioinformacional y comunicacional por excelencia, capaz de proporcionar múltiples espacios virtuales para todo tipo de usuarios que intercambian información en un mundo libre de barreras, por lo que, además, es utilizada para la realización de diversas tareas que van desde actividades personales, académicas, empresariales hasta actividades de ocio, convirtiéndose en una herramienta imprescindible para establecer la comunicación entre los seres humanos de la sociedad actual.

Internet ha permitido acortar distancias, centralizar gran cantidad de datos e información, integrar grupos afines en redes sociales. Precisamente por estas condiciones, las TIC han encontrado un escenario fundamental para su desarrollo y uso, más allá de fronteras geográficas, políticas y religiosas. Es así como dichas herramientas se han puesto en manos de todo tipo de público (niños, jóvenes, adultos), con diversa formación académica y con niveles variados de formación en uso de las TIC. 
Desde este escenario, las TIC, sin duda, han cambiado la forma de pensar, actuar y sentir de la sociedad, especialmente a partir de los años 90. Con respecto a esta nueva sociedad, Barbero (2002) afirma que la sociedad de la información y el conocimiento estimulan la innovación como resultado de la creatividad en la que se establecen sinergias en todos los campos de la acción humana. En este sentido, la información adquiere un valor importante como insumo para las actividades centrales de esta nueva sociedad que demanda oportunidad, celeridad y veracidad en los flujos informacionales.

Para ejemplificar mejor esta situación, es preciso tener en cuenta la forma como se ha transformado la dinámica de los hogares y las interacciones entre sus miembros con la llegada de los computadores, los celulares, las consolas de juegos y el Internet, entre otros. No queda duda de que los niños y jóvenes incorporan, a una velocidad extrema, las tecnologías a su vida cotidiana y hacen uso permanente de estas para la realización de diferentes actividades de entretenimiento y académicas, reflejándose en todos los sectores de la sociedad (político, económico, social).

Sin embargo, la incorporación de las TIC en los ambientes educativos no se compara con la velocidad de apropiación y uso que hacen los niños y los jóvenes de ellas en su vida diaria, ya que de acuerdo con las transformaciones sociales de la nueva sociedad se hace necesario que las TIC contribuyan a la dinamización de los procesos de enseñanza y aprendizaje en los diferentes niveles educativos, dinamización que debe contribuir a la comprensión de las nuevas formas en las que el conocimiento puede producirse, difundirse e incorporarse.

Actualmente, las instituciones de Educación Superior brindan mayor oportunidad de capacitación a la sociedad en diferentes áreas y especialidades, lo que implica cambiar la forma como han venido realizando sus procesos de enseñanza y aprendizaje y, para ello, proponen diferentes ambientes, entre ellos, la educación virtual a la que Loaiza (2002) atribuye la puesta en escena de diferentes metodologías para mediar el aprendizaje, superando las limitaciones geográficas y distribución del tiempo disponible del docente para atender las inquietudes de los estudiantes. La educación virtual, a juicio de Unigarro (2004), permite la utilización de tiempos sincrónicos o asincrónicos sin que los diferentes actores del proceso de enseñanza y aprendizaje se encuentren presentes, optimizando los recursos disponibles. El estudiante, en estas condiciones, puede acceder a procesos formativos diversos y personalizados, con la posibilidad de disponer eficientemente de su tiempo, asimilar aprendizajes a su ritmo, y plantear libremente inquietudes y opiniones que le permitan el desarrollo de sus potencialidades.

Dentro de la educación virtual existen dos tipos de estudiantes: el primero identificado como nativo digital ${ }^{1}$ que se caracteriza por haber nacido y crecido rodeado de herramientas tecnológicas (asociadas a Internet) y se destaca por absorber rápidamente la información de imágenes y vídeo como si fuese texto, consume datos de múltiples fuentes que lo hacen ágil en el manejo de gran cantidad de información y creación de contenidos, con capacidad de realizar varias tareas al mismo tiempo; sus relaciones interpersonales se centran en las redes sociales convirtiéndose en el fenómeno de la generación actual, ya que comparte información de forma natural. En cuanto al segundo tipo de estudiante identificado como inmigrante digital, este se caracteriza porque utiliza las tecnologías acorde con sus necesidades casi siempre con fines netamente laborales y/o académicos, con acercamientos tecnológicos pausados según su grado de asimilación tecnológica ya que ha debido cambiar los medios manuales por los digitales e incorporarse de alguna forma a los ambientes mediados por las TIC.

Teniendo en cuenta la heterogeneidad de los estudiantes virtuales (nativos e inmigrantes digitales), las instituciones de Educación

\footnotetext{
Los términos de nativo e inmigrante digital fueron usados por Prensky, M. (2008). En el documento Nativos e Inmigrantes Digitales. Recuperado el 03 de abril de 2012, de: http://www.nobosti.com/spip.php?article44
} 
Superior que han incorporado las TIC en los procesos de enseñanza y aprendizaje deben establecer estrategias pedagógicas, que permitan la realización de nivelaciones para disminuir las brechas tecnológicas, asegurando en los estudiantes la adquisición de competencias sobre el manejo de dichas infraestructuras tecnológicas (máquinas de cómputo, redes de comunicaciones y software con potencialidad para ofrecer gran variedad de herramientas), con el fin de que estas no se conviertan en barreras que puedan afectar sus procesos educativos.

No queda duda de que el estudiante virtual desarrolla un rol importante dentro del proceso de enseñanza y aprendizaje mediado por las TIC, ya que se convierte en protagonista de su propio aprendizaje, presentando avances que son resultado de su autodisciplina, en el que desarrolla capacidades para auto-dirigir su proceso de formación y alcanzar las metas trazadas.

\section{El rol del estudiante en la educación virtual}

En relación con el rol del estudiante en los ambientes educativos mediados por las TIC, Escudero (1992) señala que la definición de los criterios, los valores y los intereses al adoptar las TIC debe obedecer a una planificación que permita desarrollar los procesos pedagógicos, en los que el estudiante virtual desempeña un rol central representado en el conjunto de comportamientos y normas que este debe asumir como actor del proceso educativo.

Algunas características del rol del estudiante virtual que le permiten la generación del conocimiento están directamente relacionadas con la capacidad de autogestión, expresada en la autodisciplina, el autoaprendizaje, el análisis crítico y reflexivo, así como en el trabajo colaborativo, fundamental para contribuir al desarrollo del ser en su interacción y aporte con y para otros desde una mirada ética que le permite tomar conciencia de las consecuencias que pueden generar sus acciones.

A continuación, se desarrollan los roles del estudiante que, particularmente, se despliegan en el contexto de la educación virtual, dadas las características de las interacciones:
El rol del estudiante orientado al fortalecimiento de la autodisciplina. Potencia la capacidad para distribuir su tiempo, permite libertad y flexibilidad para el aprovechamiento del aprendizaje mediado por las TIC, conduciendo a la generación de movimientos de los sujetos hacia el logro de sus propias metas. La autodisciplina (Alfie Kohn, 2008) "se puede definir como el control de la propia fuerza de voluntad para cumplir cosas que generalmente se ven como deseables", lo que requiere para la gestión del aprendizaje, una dedicación permanente en las tareas propuestas, fundamentada en la definición de objetivos, su ejecución, seguimiento y control que, acompañados por la decisión, la motivación y la perseverancia, lo conducen hacia el logro de sus metas.

El rol del estudiante orientado al mejoramiento del auto aprendizaje. Definido según Rugeles, Mora, Metaute (2013) como la capacidad que desarrolla el individuo para aprender de manera autónoma, activa y participativa, adquiriendo conocimiento y habilidades y fomentando sus propios valores, lo que da como resultado la autoformación del sujeto. Es así como el autoaprendizaje le facilita al estudiante virtual el desarrollo de su capacidad de exigirse a sí mismo, lo que lo involucra en la toma de decisiones como por ejemplo en la distribución de tiempos, la ubicación de espacios, las fuentes de consulta entre las cuales se pueden mencionar bases de datos virtuales especializadas tanto libre como licenciadas, blogs, redes académicas. De igual forma, el auto aprendizaje favorece los ritmos de estudio y profundización en temas de interés, y lleva al actor a ser autodidacto y auto reflexivo. En el mismo sentido, Gisbert $(1999,56)$ asegura que "el uso de la tecnología nos permite desarrollar nuevas formas de enseñanza y aprendizaje más activas y participativas, a la vez que intuitivas y visuales que favorecen claramente los procesos de auto aprendizaje".

El rol del estudiante orientado al fortalecimiento del análisis crítico y reflexivo. Definido por Rugeles, Mora, Metaute, (2013) como la habilidad para razonar, analizar y argumentar hechos o acciones que facilitan el desarrollo integral del 
estudiante y la generación de conocimiento. El análisis crítico y reflexivo busca hacer de la educación un proceso más humanizante desde el punto de vista de la reivindicación de los actores del proceso educativo (estudiante, facilitador) como sujetos pensantes, actuantes, creadores y constructores de saberes individuales y sociales. Dicha educación humanizadora comprende las dimensiones reflexiva y crítica y, a la vez, ética, donde el pensamiento reflexivo (Escobar, Garcés, 2008) permite la comprensión de lo que aparece en el texto en relación con las experiencias y vivencias del estudiante, en lo relacionado al pensamiento crítico; para Martínez, Pascual, (2013) es un proceso cognoscitivo que toma la información, la analiza y está en capacidad de aplicarla en diversos aspectos de la vida. En el aspecto ético, Franco (2006) lo define como área del conocimiento humano que permite un discernimiento sobre el alcance de las acciones y comportamientos individuales o colectivos relacionados con los aportes constructivos o destructivos para sí, los demás y la naturaleza.

Desde el punto de vista de Lipman (1998), estas dimensiones hacen que el facilitador y el estudiante dejen de ser mediadores pasivos entre la teoría y la práctica, para convertirse en mediadores activos que, desde el hacer, reconstruyan críticamente su propia teoría y participen en el desarrollo significativo del conocimiento.

Por lo tanto, el análisis crítico y reflexivo, en el estudiante virtual, deberá ser asumido como una dinámica constante, inmersa en los procesos de enseñanza y aprendizaje, que le permita generar conocimiento aplicado, que en el marco de concepciones éticas de integralidad del ser, se convierta en experiencias significativas.

\section{El rol del estudiante orientado al} mejoramiento del trabajo colaborativo. Pretende romper el aislamiento entre los diferentes actores (Rugeles, Mora, Metaute, 2013), permitiendo ver las cualidades individuales que son compartidas entre pares y facilitadores a través de herramientas como el foro, el correo electrónico, las salas de conversación, los OVA (objetos virtuales de aprendizaje), el chat y el almacenamiento en la nube. En este sentido, Castells (2001) afirma que las personas elaboran sus redes (on line y off line) de acuerdo con sus intereses, valores, afinidades y proyectos, debido a la flexibilidad y al poder de comunicación de Internet, lo cual es fundamental en los procesos de carácter colaborativo en los ambientes educativos mediados por las TIC.

Para el estudiante virtual, el trabajo colaborativo es un factor potenciador del verdadero intercambio de conocimiento, enriquecido con las experiencias del otro, para fortalecer los aprendizajes individuales y el desarrollo de habilidades comunicativas, así como para el desarrollo de destrezas que le permiten ser más recursivo, autónomo y proactivos para la toma de decisiones. El estudiante percibe el trabajo colaborativo como aporte al desarrollo personal, respetando la diferencia y siendo tolerante, pero estableciendo lineamientos definidos con metas comunes.

De igual forma, el trabajo colaborativo le permite exponer su punto de vista a través de posiciones críticas que serán fortalecidas por sus pares y facilitadores, con el fin de buscar intercambio de conocimiento que se pueda poner a disposición de sí mismo y de los demás.

De lo anterior se deduce que el rol del estudiante en los procesos de aprendizaje bajo modalidad virtual con el apoyo de las TIC lo identifica como un sujeto activo, autogestor de sus procesos de enseñanza y aprendizaje con alto compromiso de responsabilidad frente al desarrollo de actividades relacionadas con su formación académica, personal y profesional, con capacidad de optimizar el tiempo y los recursos a su alcance teniendo en cuenta que, en lo relacionado con las TIC, deberá actualizarse permanentemente.

\section{Conclusiones}

Resulta indispensable que las instituciones de Educación Superior, cuya formación se encuentre soportada en ambientes virtuales de aprendizaje, generen estrategias pedagógicas que se orienten a la formación personal, académica y profesional, tanto para los 
nativos como para los inmigrantes digitales, reconociendo sus individualidades y ritmos de aprendizaje.

Es así como la implementación de las TIC, en relación con la Educación, no radica solo en la dotación de infraestructura tecnológica, sino en el aprovechamiento de dichas herramientas con fines académicos y sociales, especialmente, en la dinamización de los procesos educativos que puedan contribuir efectivamente con la generación y transferencia social del conocimiento, para mejorar las capacidades individuales y colectivas que puedan promover el desarrollo humano y social.

Las TIC, como mediadoras del proceso de enseñanza y aprendizaje, sin duda, han generado un gran avance en los diferentes ambientes, permitiendo utilizar de forma libre todo tipo de contenidos digitales, en los que el estudiante virtual debe asumir una actitud reflexiva desde el punto de vista de su libertad con respecto a la de los demás, para lograr ser el sujeto que bajo reflexiones éticas genere preocupaciones profundas sobre las consecuencias que tienen su propias acciones para la configuración de la sociedad.

Por lo tanto, el estudiante virtual se convierte en sujeto activo de su propio aprendizaje cuyos roles, representados en autodisciplina, auto aprendizaje, análisis crítico y reflexivo, y trabajo colaborativo, permiten que su proceso educativo sea más humanizante, como un sujeto que piensa, actúa, crea y construye saberes personales y sociales.

\section{Referencias bibliográficas}

- Alfie Kohn, (2008). Por qué está sobrevalorada la autodisciplina. Recuperado de http://www. alfiekohn.org/teaching/autodisciplina.htm.

- Barbero, Jesús M. (2002). Transformaciones del saber y del hacer en la sociedad contemporánea. Recuperado de http://www. sinectica.iteso.mx/assets/files/articulos/21_ transformaciones_del_saber_y_del_hacer_en_ la_sociedad_contemporanea.pdf, Sinéctica.

- Castells, M. (2001). La galaxia Internet. Reflexiones sobre Internet, empresa y sociedad. Barcelona: Plaza \& Janés
- Diccionario filosófico. (2012). Portal de Filosofía, Psicología y Humanidades Torre de Babel Ediciones. Recuperado de http:// www.e-torredebabel.com/Biblioteca/Voltaire/ Diccionario-Filosofico-Voltaire.htm.

- Escobar, Garcés (2008), La educación virtual y la experiencia reflexiva. Recuperado de http://revistavirtual.ucn.edu.co/index.php/ RevistaUCN/article/download/142/272.

- Escudero, Juan. (1992). La integración escolar de las nuevas tecnologías de la información. Infodidac, Revista de Informática y Didáctica.

- Franco, Zoyla. (2006). La evaluación Ética en la educación para el desarrollo humano, ISBN 958-8231-88-4, Universidad de Caldas, recuperado de https://books.google.com.co/ books?isbn=9588231884 .

- Gisbert, M. (1999). Las tecnologías de la información y la comunicación como favorecedoras de los procesos de autoaprendizaje y formación permanente, Universidad Rovira y Virgili. Revista Educar. Recuperado de: http://ddd.uab.cat/pub/ educar/0211819Xn25p53.pdf.

- Lamarca, María. (2011), Hipertexto: El nuevo concepto de documento en la cultura de la imagen. Historia de la www. Recuperado de: http://www.hipertexto.info/documentos/h_www. $\mathrm{htm}$

- Lipman, Matthew. (1998). Pensamiento complejo y educación. Madrid. Ediciones de La Torre.

- Loaiza, Roger. (2002). Facilitación y Capacitación Virtual en América Latina. Colombia

- Martínez, Pascual. (2013). La influencia de la enseñanza virtual sobre el pensamiento crítico de profesores en formación. Revista Currículum y formación del profesorado. Recuperado de: http://www.ugr.es/ recfpro/rev173COL6.pdf

- Piscitelli, Alejandro (2012). Las TIC No son una caja de herramientas, son una nueva cultura. Red Latinoamericana Portales Educativos (Relpe). Recuperado de http://www.relpe.org/ especial-del-mes/las-tic-no-son-una-caja-deherramientas-son-una-nueva-cultura/

- Piscitelli, Alejandro. (2002) Cibercutluras 2.0. En la era de las máquinas inteligentes. Paidós, Contextos. Buenos Aires Argentina.

- Piscitelli, Alejandro. (2009). Nativos Digitales. Dieta Cognitiva, Inteligencia colectiva $y$ arquitecturas de participación: Santillana. 
- Prensky, M. (2008). Nativos e inmigrantes digitales. Recuperado de http://www.nobosti. com/spip.php?article44

- Rugeles, P.; Metaute, P. y Mora, B. (2013). Caracterización de experiencias significativas mediadas por las TIC en educación superior virtual. Global Conference on Business \& Finance Proceedings. 8 (2). P 1485-1494. Recuperado de: http://eds.b.ebscohost.com/ eds/detail/detail ?vid=7\&sid=4a1e7119-a43f4b67-b1c6-6123b01085dc\%40sessionmgr110 \&hid=112\&bdata=Jmxhbmc9ZXMmc2I0ZT1IZH MtbGI2ZQ\%3d\%3d\#db=bth\&AN=89496982.
- Rugeles, P.; Metaute, P. y Mora, B. (2014), El trabajo colaborativo en la educación superior mediada por las Tecnologías de Información y Comunicación. Global Conference on Business \& Finance Proceedings. 2014.9 (1) Recuperado de: http://eds.b.ebscohost.com/eds/pdfviewer/ pdfviewer?vid=10\&sid=4a1e7119-a43f-4b67b1c6-6123b01085dc\%40sessionmgr110\&h $\mathrm{id}=112$

- Unigarro, Manuel. (2004). Educación Virtual: Encuentro formativo en el ciberespacio. Editorial: UNAB. Bucaramanga. 